\title{
Autoren dieses Heftes
}

Thomas Blanke

Lorenz Böllinger

Ute Daub

Rainer Eckertz

Tilman Evers

Günter Jerouschek

Ingeborg Maus

Joachim Nocke

Christoph Schmedt

Heinz Eduard Tödt

Gert Winter

Rainer Wolf geb. 1944, Dr. jur., Professor für Arbeitsrecht an der Universität Oldenburg, Werbachstr. 34, 2900 Oldenburg geb. 1944, Dr.jur., Dipl.Psych., psychoanalytische Weiterbildung DPV, Professor für Kriminologie und Strafrecht an der Universität Bremen, Eichendorffstr. 27, 6000 Frankfurt am Main I

geb. 1947, Diplomsoziologin und Publizistin, Brükkenstr. 34, 6000 Frankfurt am Main 70

geb. 1944, Dr. jur., Richter, korrespondierendes Mitglied der Forschungsstätte der Ev. Studiengemeinschaft (Heidelberg), Keltenweg 4, 69is Dossenheim

geb. I942, Dr. jur., Privatdozent für Politische Wissenschaft und Soziologie an der FU Berlin, Studienleiter an der Evangelischen Akademie Hofgeismar, Postfach I 205, 3520 Hofgeismar

geb. I950, Dr. jur., M. A., Hochschulassistent, Universität Hannover, Hanomagstr. 8, 3000 Hannover 9I

Dr. phil., Privatdozentin am Fachbereich Gesellschaftswissenschaft der Universität Frankfurt am Main, Parkstr. 5, 6000 Frankfurt am Main I

geb. 1942, Dr. jur., Akademischer Oberrat an der Universität Hannover, Hanomagstr. 8, 3000 Hannover 9I

geb. 1955, wissenschaftlicher Mitarbeiter am Lehrgebiet für Zivil- und Arbeitsrecht der Universität Hannover, Hanomagstr. 8, 3000 Hannover 9I

geb. I918, Dt. theol., em. Professor für Systematische Theologie und Sozialethik an der Universität Heidelberg, Schloßwolfsbrunnenwg 20, 6900 Heidelberg

geb. 1943, Dr. jur., Professor für Öffentliches Recht und Rechtssoziologie an der Universität Bremen, Elsa-Brandström-Str. 8, 2800 Bremen

geb. 1950, Dr. jur., Regierungsrat beim Minister für Stadtentwicklung, Wohnen und Verkehr des Landes Nordrhein

- Westfahlen, Drake Platz I, 4000 Düsseldorf 